\title{
The Parameterized Complexity of Centrality Improvement in Networks *
}

\author{
Clemens Hoffmann ${ }^{1}$, Hendrik Molter ${ }^{1}$, and Manuel Sorge ${ }^{2}$ \\ ${ }^{1}$ Institut für Softwaretechnik und Theoretische Informatik, TU Berlin, Germany, \\ h.molter@tu-berlin.de \\ ${ }^{2}$ Dept. Industrial Engineering and Management, Ben-Gurion University of the Negev, \\ Beer Sheva, Israel, sorge@post.bgu.ac.il
}

July 11, 2018

\begin{abstract}
The centrality of a vertex $v$ in a network intuitively captures how important $v$ is for communication in the network. The task of improving the centrality of a vertex has many applications, as a higher centrality often implies a larger impact on the network or less transportation or administration cost. In this work we study the parameterized complexity of the NP-complete problems Closeness Improvement and Betweenness ImproveMENT in which we ask to improve a given vertex' closeness or betweenness centrality by a given amount through adding a given number of edges to the network. Herein, the closeness of a vertex $v$ sums the multiplicative inverses of distances of other vertices to $v$ and the betweenness sums for each pair of vertices the fraction of shortest paths going through $v$. Unfortunately, for the natural parameter "number of edges to add" we obtain hardness results, even in rather restricted cases. On the positive side, we also give an island of tractability for the parameter measuring the vertex deletion distance to cluster graphs.
\end{abstract}

\section{Introduction}

Measuring the centrality of a given vertex in a network has attracted the interest of researchers since the second half of the 20th century [13], see Newman's book [19] for an overview. There are various interpretations of what makes a vertex more central than another vertex in a network. Two popular measures for the centrality of a vertex $z$ are closeness centrality $c_{z}$ and betweenness centrality $b_{z}$ [13]. They are based on the distances of the given vertex $z$ to the remaining vertices and on the number of shortest paths going through $z$, respectively. For this work, we use the following definitions.

$$
c_{z}=\sum_{\substack{u \in V \\ d(u, z)<\infty \\ u \neq z}} \frac{1}{d(z, u)} \quad b_{z}=\sum_{\substack{s, t \in V \\ s \neq t ; s, t \neq z \\ \sigma_{s t} \neq 0}} \frac{\sigma_{s t z}}{\sigma_{s t}}
$$

${ }^{*}$ MS supported by the People Programme (Marie Curie Actions) of the European Union's Seventh Framework Programme (FP7/2007-2013) under REA grant agreement number 631163.11 and the Israel Science Foundation (grant no. 551145/14). 
Herein, $d(s, t)$ is the distance between two vertices $s$ and $t$, that is, the number of edges on a shortest $s-t$ path, $\sigma_{s t}$ is the number of shortest $s$ - $t$ paths, and $\sigma_{s t z}$ is the number of shortest $s-t$ paths that contain $z$. Intuitively, if $z$ has many close-by vertices, then its closeness centrality is large, and if $z$ is on shortest paths between many vertices, then its betweenness centrality is large. The closeness centrality as defined above is also known as the harmonic centrality 1$]$

Analyzing vertex centrality in networks has been studied intensively (e.g. [5, 13, 19, 20, 21]) and comprises a diverse set of applications in, e.g., biological [24], economic [22], and social networks [13]. Some examples: A transport company might be interested in placing its depots centrally such that the transportation costs are rather low. The value of an airport might be influenced by its centrality in the flight-connection network between airports. The most central nodes in a computer network may be useful for determining the locations of data centers where the routes are short and peering costs are low. In social networks, economically important influencers are presumably more central than other users.

Since it is so desirable to find vertices with large centrality in a graph, vertices have incentive to improve their own centrality. E.g., a social network member might want to increase her impact on other users by increasing her own centrality, or an airport operator wants to increase the appeal of her airport for investors (as measured by the centrality). In both cases, natural operations are to introduce new links into the network, i.e., to make new acquaintances or incentivise airlines to offer certain routes. In this work, we hence study the complexity of improving the centrality of a given vertex by introducing new links into the network. Formally, the computational problems that we study are defined as follows.

Closeness (Betweenness) Improvement

Input: $\quad$ An undirected, unweighted graph $G=(V, E)$, a vertex $z \in V$, an integer $k$ and a rational number $r$.

Question: Is there an edge set $S, S \cap E=\emptyset$, of size at most $k$ such that $c_{z} \geq r$ $\left(b_{z} \geq r\right)$ in $G+S:=(V, E \cup S)$ ?

We also say that an edge set $S$ as above is a solution.

The above two problems were introduced by Crescenzi et al. [6] and D'Angelo et al. [9], respectively, who gave approximation algorithms and showed that their empirical approximation ratios are close to one on random graphs with up to 100 vertices and up to 1000 vertices, respectively. In a corresponding presentation Crescenzi et al. [6] noted that finding the optimal solution for comparison was very time consuming. Here, we study the parameterized complexity of Closeness Improvement and Betweenness Improvement with the ultimate goal to design efficient exact algorithms. That is, we aim to find fixed-parameter (FPT) algorithms with running time $f(k) \cdot n^{O(1)}$, where $n$ is the input length and $k$ is some secondary measure, called parameter, or we show $\mathrm{W}[1]$ or $\mathrm{W}[2]$-hardness, meaning that there are presumably no FPT algorithms.

Our Results. Our results for Closeness Improvement are as follows. From two reductions from Dominating SeT it follows that Closeness Improvement is NP-hard on (disconnected) planar graphs with maximum degree 3 and $\mathrm{W}[2]$-hard with respect to $k$, the number of added edges, on disconnected split graphs, for example (Corollary 2). Split graphs are a simple model of core-periphery structure, which occurs in social and biological networks [7]. In particular, we can derive that a straightforward $n^{O(k)}$-time algorithm for Closeness ImProvement is asymptotically optimal. Motivated by the fact that social networks often have small diameter

\footnotetext{
${ }^{1}$ There are several definitions for closeness centrality in the literature. We use the present one because it is natural [19] and it was used in closely related work [6].
} 
in conjunction with small H-index [1], we show that CLOSENESS IMPROVEMENT remains NPhard on (connected) graphs of diameter at most 6 and H-index 4 (Theorem 2). On the positive side, we show that ClOSENESS IMPROVEMENT allows a fixed-parameter algorithm with respect to the parameter distance to cluster graph, that is, the smallest number of vertices to delete in order to obtain a cluster graph. Directed Closeness Improvement is NP-hard and $\mathrm{W}[2]$-hard with respect to $k$ even if the input graph is acyclic (Theorem 4) or has diameter 4 (Theorem 5).

For Betweenness Improvement the picture is similar. It is W[2]-hard with respect to $k$ (Theorem 6) also in the directed case (Theorem 8), NP-hard for graphs of H-index 4 (Corollary 5), and Betweenness Improvement is fixed-parameter tractable with respect to $k$ and the distance to cluster graph combined.

Preliminaries and Notation. We use standard notation from graph theory [23]. Throughout, we refer to the number of vertices as $n$ and to the number of edges ( $\operatorname{arcs}$ ) as $m$. For two vertices $u, v$ we denote by $d(u, v)$ the distance between $u$ and $v$, i.e. the number of edges on a shortest path from $u$ to $v$. If $u$ and $v$ are not connected by a path, then $d(u, v)=\infty$. A split graph allows for a partition of the vertex set into a clique and an independent set. In a cluster graph each connected component is a clique. The diameter of a graph is $\infty$ if it is disconnected and the maximum distance of any two vertices otherwise. The $H$-index of a graph is the largest integer $h$ such that there $h$ vertices of degree at least $h$.

We also use standard notation from parameterized complexity [8]. Importantly, a parameterized reduction from a parameterized problem $L \subseteq \Sigma^{*} \times \mathbb{N}$ with parameter $k$ to a parameterized problem $L^{\prime} \subseteq \Sigma^{*} \times \mathbb{N}$ with parameter $k^{\prime}$ is a $g(k) \cdot|I|^{\mathcal{O}(1)}$-time computable function $f: \Sigma^{*} \times \mathbb{N} \rightarrow \Sigma^{*} \times \mathbb{N}:(I, k) \rightarrow\left(I^{\prime}, k^{\prime}\right)$ such that $k^{\prime} \leq h(k)$ for some computable function $h$ and $(I, k) \in L \Leftrightarrow\left(I^{\prime}, k^{\prime}\right) \in L^{\prime}$.

The Exponential Time Hypothesis roughly states that satisfiability of a Boolean formula in conjunctive normal form with clauses of size 3 cannot be decided in $2^{o(n)}$ time, see Impagliazzo and Paturi [16], Impagliazzo et al. [17] for details.

\section{Closeness Centrality}

In this section, we present algorithmic and hardness results for Closeness Improvement. First, we make an important observation that will help us in our proofs. Intuitively, we show that to improve the closeness of a vertex by adding edges, it always makes sense to add only edges adjacent to that vertex.

Lemma 1. Let $I=(G=(V, E), z, k, r)$ be a Closeness Improvement instance. If $I$ is a YES-instance, then $c_{z}$ can be increased to $r$ by adding at most $k$ edges, all of which contain $z$.

Proof. Let $u, v \in V \backslash\{z\}$ be two vertices of the input graph $G$ such that $e:=\{u, v\} \notin E$. Assume for the sake of contradiction that $S$ is a solution with the largest number of edges incident to $z$ and that $\{u, v\} \in S$. Consider the shortest paths from $u$ to $z$ and from $v$ to $z$ in $G+S$. If these paths have the same length, then neither contains $\{u, v\}$. Hence, in this case we have that $c_{z}$ in $G+S$ equals $c_{z}$ in $G+(S \backslash\{u, v\})$. Thus, adding an arbitrary edge to $(S \backslash\{u, v\}$ yields a solution with a greater number of edges incident to $z$ than $S$, a contradiction. Hence, one of the two shortest paths is shorter than the other; say $u$ is closer to $z$ than $v$. (Observe that, hence, $\{v, z\} \notin E$.) In this case, $c_{z}$ in $G+((S \backslash\{u, v\}) \cup\{v, z\})$ is at least as large as $c_{z}$ in $G+S$ : Consider any shortest path $P$ in $G+S$ from some vertex $w$ to $z$ that contained $\{u, v\}$. Since $u$ is closer to $z$ than $v$, path $P$ contains first $v$ and then $u$. Hence, replacing the remaining path after $v$ with the direct edge to $z$ shortens $P$. Hence, $((S \backslash\{u, v\}) \cup\{v, z\})$ contains more 

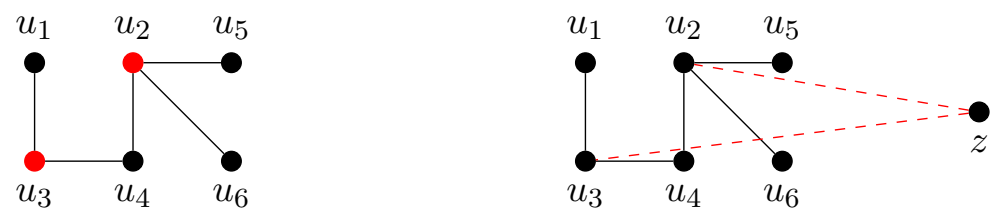

Figure 1: Reduction from Dominating Set to Closeness Improvement. Left: A DomiNATING SET instance $I=(G, k=2)$. The red colored vertices form a solution for $I$. Right: The constructed Closeness Improvement instance $\left(I^{\prime}=G^{\prime}, z, k^{\prime}=2, r=k+\frac{n-k}{2}\right)$. The red dashed edges form a solution for $I^{\prime}$.

edges incident to $z$ and yields $c_{z}$ which is at least large as for $S$, a contradiction. Hence, the solution with the largest number of edges incident to $z$ does not contain any edges not incident to $z$, showing the lemma.

From this observation follows, that if we were to try all possibilities to solve Closeness IMPROVEMENT, it suffices to consider only sets of edges to add, where every edge is adjacent to the node whose closeness we want to improve. Hence, we get an XP algorithm with respect to $k$.

Corollary 1. Closeness Improvement is solvable in $O\left(n^{k} \cdot(n+m)\right)$ time where $k$ is the number of edge additions, and thus is in XP with respect to the parameter number $k$ of edge additions.

Hardness Results. Next, we present several hardness results for Closeness Improvement: They are based on two reductions from Dominating SET; a simple one and a more intricate one. From results on Dominating SET we can then infer corresponding results for Closeness Improvement. In particular, we show that the $n^{O(k)}$-time algorithm from Corollary 1 is essentially optimal unless the Exponential Time Hypothesis is false.

Theorem 1. Closeness Improvement is NP-hard and W[2]-hard with respect to the number $k$ of edge additions (on disconnected graphs). Moreover, unless the Exponential Time Hypothesis fails, Closeness ImProvement does not allow an algorithm with running time $f(k)$. $n^{o(k)}$.

Proof. We give a reduction from Dominating SET which is W[2]-hard and does not admit an algorithm with running time $f(k) \cdot n^{o(k)}$ unless the Exponential Time Hypothesis is false [8]. Let $I=(G=(V, E), k \in \mathbb{N})$ where $V=\left\{u_{1}, \ldots, u_{n}\right\}$. We construct a Closeness ImproveMENT instance $I^{\prime}=\left(G^{\prime}=\left(V^{\prime}, E^{\prime}\right), z, k, k+\frac{1}{2}(n-k)\right)$ as follows: Given the input graph $G$, we simply add an isolated vertex $z$ to the graph, that is $G^{\prime}=(V \cup\{z\}, E)$.

We now show that the reduction is correct, i.e. $I$ is a Yes-instance if and only if $I^{\prime}$ is a YES-instance:

$\Rightarrow$ : Let there be a dominating set $V_{D S} \subseteq V$ of size $k$ in $G$. After adding $k$ edges between $z$ and each vertex in $V_{D S}$ in $G^{\prime}$, these $k$ vertices have distance 1 to $z$ and the $n-k$ neighbors of the vertices $V_{D S}$ have distance 2 to $z$. Hence, $c_{z}=k+\frac{n-k}{2}$. That is, $I^{\prime}$ is a YES-instance.

$\Leftarrow$ : The reverse direction is by contraposition. Assume that there is no dominating set of size $k$ in $G$. Lemma 1 shows that we can maximally increase $c_{z}$ by adding edges where one endpoint is $z$. However, after adding $k$ edges between $z$ and $k$ other vertices in $G^{\prime}$, there are $\ell \geq 1$ vertices in $G^{\prime}$ whose distances to $z$ is $d \geq 3$. Hence, $c_{z} \leq k+\frac{n-k-\ell}{2}+\frac{\ell}{d}<k+\frac{n-k}{2}$. That is, $I^{\prime}$ is a No-instance. 

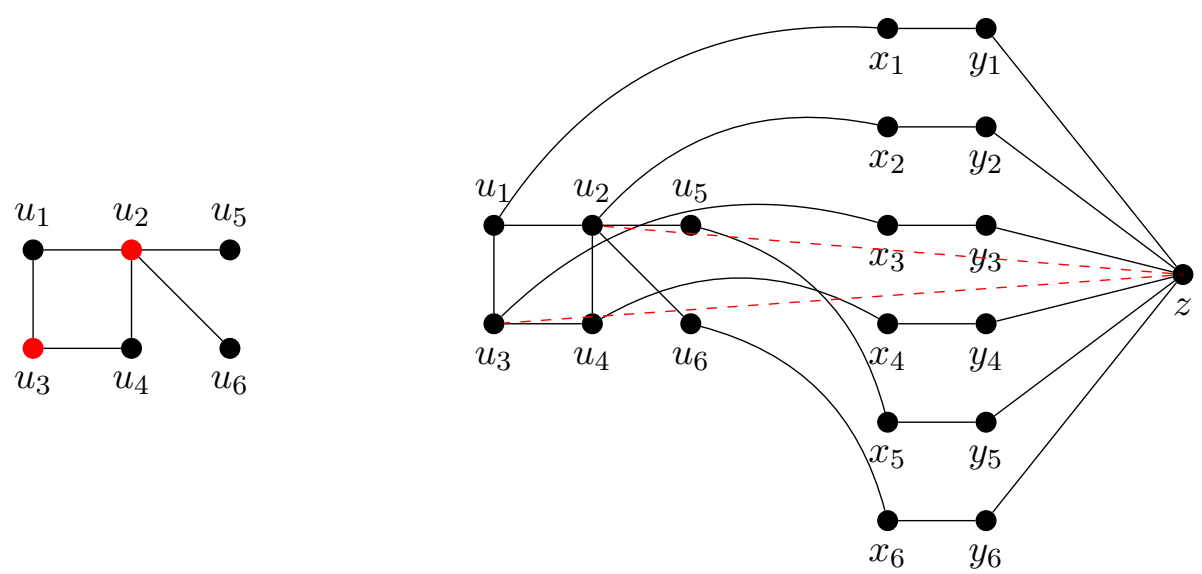

Figure 2: Parameterized reduction from Dominating Set to Closeness Improvement on graphs with diameter 4. Left: A Dominating Set instance $I=(G, k=2)$. The red colored vertices $u_{2}$ and $u_{3}$ form a solution for $I$. Right: The constructed CLOSENESS IMPRovemenT instance $\left(I^{\prime}=G^{\prime}, z, k^{\prime}=2,2 n+\frac{k}{2}\right)$. The red, dashed edges form a solution for $I^{\prime}$.

\section{Corollary 2. Closeness Improvement is}

1. NP-hard even on disconnected planar graphs with maximum degree 3,

2. NP-hard and W[2]-hard on disconnected split graphs, and

3. NP-hard and W[2]-hard on disconnected graphs in which each connected component has diameter two.

Proof. Instead of the plain Dominating SET problem, we reduce from special cases which have been shown to be hard in the literature. For the first result, we reduce from Dominating SET on planar graphs with maximum degree 3 [14]. Clearly, the reduction in Theorem 1 does not destroy planarity or increase the maximum degree.

For the second result, we use the standard reduction from Set Cover to Dominating SET in split graphs, which identifies the size of the sought set cover and the size of the sought dominating set [2]. Since SET Cover is W[2]-hard with respect to the size of the sought set cover [10], so is Dominating SET on split graphs with respect to the size of the sought dominating set. Hence, the same is true for Closeness Improvement on split graphs by the reduction in Theorem 1 .

For the third result, we use the fact that Dominating SeT is W[2]-hard on graphs of diameter 2 [18].

Applications in social networks, which have both small diameter and small H-index [2] [1], motivate the following more special hardness result where both these values are small constants. It also shows that CLOSENESS IMPRovement remains hard on connected graphs, which was left open by Theorem 1 ,

Theorem 2. Closeness IMPROVEMENT is NP-hard and W[2]-hard with respect to the parameter number $k$ of edge additions even on connected graphs with diameter 4. Moreover, ClosENESS IMPROVEMENT is NP-hard even on graphs which simultaneously have diameter 6 and H-index 4.

Proof. The proof is by a parameterized reduction from two variants of Dominating SET, detailed below. Let $I=(G=(V, E), k \in \mathbb{N})$ be a Dominating Set instance where $V=$ $\left\{u_{1}, \ldots, u_{n}\right\}$. We construct a Closeness Improvement instance $I^{\prime}=\left(G^{\prime}=\left(V^{\prime}, E^{\prime}\right), z, k, 2 n+\right.$

\footnotetext{
${ }^{2}$ Recall that the H-index of a graph is the largest integer $h$ such that there are $h$ vertices of degree at least $h$.
} 
$\frac{k}{2}$ ) as follows (see Fig. 2): Given the input graph $G$, we add $2 n$ vertices $x_{1}, \ldots, x_{n}, y_{1}, \ldots, y_{n}$ such that each vertex $x_{i}$ is adjacent to $u_{i}$ and $y_{i}$. Furthermore, we add $z$ and add edges between $z$ and each $y_{1}, \ldots, y_{n}$. Formally, $V^{\prime}=V \cup\left\{x_{i}, y_{i} \mid 1 \leq i \leq n\right\} \cup\{z\}$ and $E^{\prime}=$ $E \cup\left\{\left\{u_{i}, x_{i}\right\},\left\{x_{i}, y_{i}\right\},\left\{z, y_{i}\right\} \mid 1 \leq i \leq n\right\}$.

We partition $V^{\prime}$ into the subsets $Y^{\prime}:=\left\{y_{1}, \ldots, y_{n}\right\}, X^{\prime}:=\left\{x_{1}, \ldots, x_{n}\right\}$ and $U^{\prime}:=\left\{u_{1}, \ldots, u_{n}\right\}$. Note that the vertices in $Y^{\prime}$ have distance 1 to $z$, the vertices in $X^{\prime}$ have distance 2 to $z$ and the vertices in $U^{\prime}$ all have distance 3 to $z$. This completes the construction which can clearly be carried out in polynomial time.

Suppose the reduction is correct. To get NP-hardness and W[2]-hardness with respect to $k$ on diameter 4 graphs, we reduce from Dominating SET on graphs of diameter two, which is NP-hard [1] and W[2]-hard with respect to $k$ [18]. It is not hard to see that the resulting graph $G^{\prime}$ indeed has diameter 4 . To get NP-hardness on graphs with simultaneously diameter 6 and H-index 4, reduce instead from Dominating Set on graphs $G$ with maximum degree 4. By the connections via $x_{i}, y_{i}$, and $z$, any two vertices of $G$ are connected in $G^{\prime}$ by a path of length at most 6 . Graph $G^{\prime}$ has H-index 4 , because it has maximum degree 4 apart from $z$.

First, we show that adding edges between $z$ and vertices in $U^{\prime}$ is optimal:

Assume an edge $\left\{z, x_{i}\right\}, x_{i} \in X^{\prime}$, is added. Then the distance between $z$ and $x_{i}$ is 1 and the distance between $z$ and $u_{i}$ is 2 . If we instead add the edge $\left\{z, u_{i}\right\}$, then the distance between $z$ and $u_{i}$ is 1 and the distance between $z$ and $x_{i}$ remains 2. Furthermore, the edge $\left\{z, u_{i}\right\}$ may introduce shorter distances to the neighbors of $u_{i}$, which the edge $\left\{z, x_{i}\right\}$ does not. Hence, if a solution for $I^{\prime}$ contains $\left\{z, x_{i}\right\}$, we can replace that edge by $\left\{z, u_{i}\right\}$.

It remains to show that the reduction is correct, i.e. $I$ is a Yes-instance if and only if $I^{\prime}$ is a Yes-instance:

$\Rightarrow$ : Let $I$ be a YeS-instance. Then there is a dominating set $U_{D S}^{\prime} \subseteq U^{\prime}$ of size $k$ for $G^{\prime}-$ $\left(X^{\prime} \cup Y^{\prime}\right)$. After adding $k$ edges between $z$ and each vertex in $U_{D S}^{\prime}$, these $k$ vertices have distance 1 to $z$ and the $n-k$ neighbors in $U^{\prime} \backslash U_{D S}^{\prime}$ have distance 2 to $z$. Furthermore, each vertex in $Y^{\prime}$ has distance 2 to $z$ and each vertex in $X$ has distance 1 to $z$.

Hence, $c_{z}=k+\frac{n-k}{2}+\frac{n}{2}+n=2 n+\frac{k}{2}$. That is, $I^{\prime}$ is a Yes-instance.

$\Leftarrow$ : We prove the other way by contraposition. That is, we show that $I^{\prime}$ is a No-instance if $I$ is a No-instance. Let $I$ be a No-instance. If there is no dominating set of size $k$ in $G^{\prime}-\left(X^{\prime} \cup Y^{\prime}\right)$, then after adding $k$ edges between $z$ and vertices in $U^{\prime}$, there are $l \geq 1$ vertices in $U^{\prime}$ whose distances to $z$ is still 3 .

Hence, $c_{z}=k+\frac{n-k-l}{2}+\frac{l}{3}+\frac{n}{2}+n=2 n+\frac{k}{2}-\frac{l}{6}$, for $l \geq 1$. That is, $I^{\prime}$ is a No-instance.

We note that it is not hard to show that Closeness Improvement is polynomial-time solvable on graphs of diameter 2 . The case of diameter 3 remains open.

Algorithmic Result. Now we present an algorithm for Closeness Improvement, which shows that the problem is fixed-parameter tractable when parameterized by the distance of the input graph to a cluster graph.

Theorem 3. Closeness ImPRovement can be solved in $2^{2^{2^{O(\ell)}}} \cdot n^{O(1)}$ time, where $\ell$ is the vertex deletion distance of $G$ to a cluster graph, and thus is in FPT with respect to the parameter $\ell$.

Proof. Let $(G, z, k, r)$ be a Closeness Improvement instance, where $V_{\text {VDS }} \subset V$ is a vertex set of size $\ell$ such that $G_{C}=\left(V_{C}, E_{C}\right):=G-V_{\text {VDS }}$ is a cluster graph with the set of connected components $\mathcal{C}=\left\{C_{1}, \ldots, C_{s}\right\}$ which we also call clusters. Since a cluster vertex deletion set $V_{\text {VDS }}$ of size $\ell$ can be found in $O\left(1.92^{\ell} \cdot(n+m)\right)$ time if it exists [3, 15], we may assume that $V_{\text {VDS }}$ is given. By Lemma 1 we may assume that the edges in an optimal solution $E^{*}$ to $(G, z, k, r)$ 
all have endpoint $z$. Hence, in the following we denote by a solution $V^{\prime}$ the endpoints different from $z$ of the corresponding edge set. Any solution can thus be divided into vertices in $V_{\text {VDS }}$ and those in $V \backslash V_{\text {VDS }}$. Let $V_{\text {VDS }}^{*}$ be the intersection of an optimal solution $V^{*}$ with $V_{\text {VDS }}$. The first step in our algorithm is to find $V_{\mathrm{VDS}}^{*}$, by trying all $2^{\ell}$ possibilities. It remains to determine $V^{*} \backslash V_{\text {VDS }}$. Intuitively, if there are vertices which have the same neighborhood in $V_{\text {VDS }}$ and are in clusters that also have the same neighborhood in $V_{V D S}$, then each such vertex after the first one does not help to shorten distances to $z$ for any vertex except itself. Hence, if we know that the optimal solution contains vertices in clusters both with some specified neighborhood in $V_{\text {VDS }}$, then we can assume that these vertices are distributed among the largest clusters with that neighborhood. In the algorithm we thus first determine for which neighborhoods in $V_{\text {VDS }}$ there are clusters and vertices in these clusters in the optimal solution. Then we distribute the vertices in the solution optimally among the chosen neighborhoods. The proof that this yields an optimal solution is unfortunately technical and we need the following notation.

We say that the signature $\operatorname{sig}\left(C_{i}\right)$ of a cluster $C_{i}, i=1, \ldots, s$, is the set of neighbors in $V_{\text {VDS }} \cup\{z\}$ of vertices in $C_{i}$, that is, the signature is $\left\{v \in V_{\text {VDS }} \cup\{z\} \mid \exists u \in C_{i}:\{u, v\} \in E\right\}$. Similarly, the signature $\operatorname{sig}(v)$ of a vertex $w \in V \backslash V_{\mathrm{VDS}}$ is $N(v) \cap\left(V_{\mathrm{VDS}} \cup\{z\}\right)$. For some subset $V_{i} \subseteq C_{i}$ of some cluster $C_{i} \in \mathcal{C}$ denote by the signature $\operatorname{sig}\left(V_{i}\right)$ of $V_{i}$ the tuple $\left(\operatorname{sig}\left(C_{i}\right),\{\operatorname{sig}(v) \mid\right.$ $\left.\left.v \in V_{i}\right\}\right)$. Say also that $C_{i}$ is $V_{i}$ 's cluster. Now the signature $\operatorname{sig}(\hat{V})$ of a solution $\hat{V}$ is the set $\left\{\operatorname{sig}\left(V_{i}\right) \mid C_{i} \in \mathcal{C} \wedge C_{i} \cap \hat{V}=V_{i} \neq \emptyset\right\}$. That is, the signature of $\hat{V}$ encodes the signatures of the clusters touched by $\hat{V}$ along with, for each touched cluster, the signatures of all vertices touched by $\hat{V}$ in that cluster. Say that a vertex subset $V_{j}$ of some cluster $C_{j}$ is eligible for some signature $\operatorname{sig}\left(V_{i}\right)$ of a vertex subset $V_{i}$ of a cluster $C_{i}$ if $\operatorname{sig}\left(V_{j}\right)=\operatorname{sig}\left(V_{i}\right)$. Accordingly, for some solution $\hat{V}$ with signature $\operatorname{sig}(\hat{V})$, say that a vertex subset $V_{i} \subseteq C_{i}$ of some cluster $C_{i}$ is eligible for $\operatorname{sig}(\hat{V})$ if $\operatorname{sig}\left(V_{i}\right) \in \operatorname{sig}(\hat{V})$. Finally, the reduct of a solution $\hat{V}$ is a subset $V^{\prime} \subseteq \hat{V}$ such that, for each cluster $C_{i} \in \mathcal{C}$ with $\hat{V} \cap C_{i} \neq \emptyset$ and each vertex signature $S \in\left\{\operatorname{sig}(v) \mid v \in \hat{V} \cap C_{i}\right\}$, there is exactly one vertex $u \in V^{\prime} \cap C_{i}$ with signature $S$. Observe that, if $V^{\prime}$ is the reduct of $\hat{V}$ and $V^{\prime \prime} \supseteq V^{\prime}$ is any superset of $V^{\prime}$ with $\left|V^{\prime \prime}\right|=|\hat{V}|$, then $\operatorname{sig}(\hat{V})=\operatorname{sig}\left(V^{\prime}\right) \subseteq \operatorname{sig}\left(V^{\prime \prime}\right)$ and the closeness centrality of $z$ achieved by $V^{\prime \prime}$ is at least the one achieved by $\hat{V}$.

Let $S$ be the signature of some vertex subset of some cluster. Call a vertex subset $V_{i} \subseteq C_{i}$ of some cluster $C_{i} \in \mathcal{C}$ most potent for $S$ if it is eligible for $S$ and among all vertex subsets of some cluster in $\mathcal{C}$ that are eligible for $S$ we have that $V_{i}$ 's cluster is the largest. If the signature $S$ is clear from the context, we say that $V_{i}$ is most potent.

Let $V^{*}$ be the reduct of an optimal solution. We claim that there is an optimal solution with reduct $V_{2}^{*}$ with signature $\operatorname{sig}\left(V_{2}^{*}\right)=\operatorname{sig}\left(V^{*}\right)$ such that, for each $S \in \operatorname{sig}\left(V_{2}^{*}\right)$, there is a vertex subset $V_{j}$ of some cluster contained in $V^{*}$ that is most potent among vertex subsets eligible for $S$. Assume the claim does not hold. Then there exists the reduct $V_{3}^{*}$ of some optimal solution such that $V_{3}^{*}$ contains the largest number of most potent vertex sets and at least one signature $S \in \operatorname{sig}\left(V_{3}^{*}\right)$ such that no vertex subset of some cluster which is most potent for $S$ is contained in $V_{3}^{*}$. Observe however, that some vertex subset $V_{i} \subseteq C_{i}$ with $\operatorname{sig}\left(V_{i}\right)=S$ is contained in $V_{3}^{*}$. Let $V_{j}$ be most potent among vertex sets with signature $\operatorname{sig}\left(V_{i}\right)$ and let $V_{4}^{*}=\left(V_{3}^{*} \backslash V_{i}\right) \cup V_{j}$. Note that, $\operatorname{sig}\left(V_{4}^{*}\right)=\operatorname{sig}\left(V_{3}^{*}\right)$ and, because of that, each vertex in $V \backslash\left(C_{i} \cup C_{j}\right)$ has the same distance to $z$ according to $V_{3}^{*}$ and to $V_{4}^{*}$. However, since $\left|C_{i}\right|<\left|C_{j}\right|, \operatorname{sig}\left(V_{i}\right)=\operatorname{sig}\left(V_{j}\right)$, and since, for each vertex signature in $\operatorname{sig}\left(V_{i}\right)$ there is at most one vertex in each of $V_{4}^{*}$ and $V_{3}^{*}$ with that signature, more vertices have distance 2 to $z$ according to $V_{4}^{*}$ than to $V_{3}^{*}$. This is a contradiction to $V_{3}^{*}$ being the reduct of an optimal solution. Hence, the claim holds. Thus, once we know the signature of an optimal solution, we know it is optimal to take the most potent (according to that signature) vertex sets into our solution.

Let $V^{*}$ again be the reduct of an optimal solution. The remainder of $V^{*}$ is the subset of $V^{*}$ resulting from removing for each $S \in \operatorname{sig}\left(V^{*}\right)$ a most potent vertex set $V_{j}$ with signature $\operatorname{sig}\left(V_{j}\right)=$ 

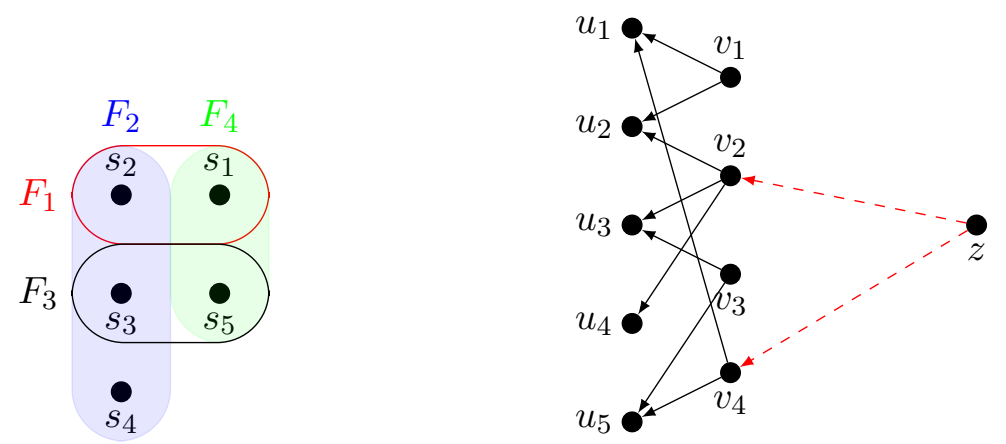

Figure 3: Parameterized reduction from Set Cover to Directed Closeness Improvement. Left: A Set Cover instance $I=(U, \mathcal{F}, k=2)$ with solution $\left\{F_{2}, F_{4}\right\}$. Right: The constructed Directed Closeness Improvement instance $I^{\prime}=\left(G, z, k=2, r=4 \frac{5}{6}\right)$. The red dashed edges imply a solution for $I^{\prime}$.

$S$ from $V^{*}$ (note that the $V_{j}$ 's are present without loss of generality by the previous claim).

We claim that there is some optimal solution with reduct $V_{2}^{*}$ with signature $\operatorname{sig}\left(V_{2}^{*}\right)=$ $\operatorname{sig}\left(V^{*}\right)$ such that the remainder of $V_{2}^{*}$ contains among all vertex subsets of some cluster with a signature in $\operatorname{sig}\left(V_{2}^{*}\right)$ those vertex subsets in the largest clusters. Assume otherwise. Then there exists the reduct $V_{3}^{*}$ of some optimal solution such that the remainder $V_{3}^{*}, R$ of $V_{3}^{*}$ contains some $V_{i} \subseteq C_{i}$ such that $V_{3}^{*, R} \cap C_{i}=V_{i}$, and there is a cluster $C_{j}$ and a vertex subset $V_{j} \subseteq C_{j}$ such that $V_{3}^{*, R} \cap V_{j}=\emptyset, \operatorname{sig}\left(V_{j}\right), \operatorname{sig}\left(V_{i}\right) \in \operatorname{sig}\left(V_{3}^{*}\right)$, and $\left|C_{j}\right|>\left|C_{i}\right|$. Let $V_{4}^{*}=\left(V_{3}^{*} \backslash V_{i}\right) \cup V_{j}$. Note that $\operatorname{sig}\left(V_{3}^{*}\right)=\operatorname{sig}\left(V_{4}^{*}\right)$ and, because of that, each vertex in $V \backslash\left(C_{i} \cup C_{j}\right)$ has the same distance to $z$ according to $V_{3}^{*}$ and to $V_{4}^{*}$. By the same argument as in the previous claim, we obtain a contradiction to $V_{3}^{*}$ being the reduct of an optimal solution. Hence, also this claim holds. Thus, once we know the signature $S$ of an optimal solution, we know it is optimal to take into the remainder of the optimal solution those vertex subsets with a signature in $S$ that are contained in the largest clusters.

The algorithm to compute an optimal solution $V^{*}$ is now as follows. Try all possibilities for the intersection $V^{*} \cap V_{\text {VDS }}$. Next, try all possibilities for the signature $S$ of $V^{*}$. Put into $V^{*}$, for each $S^{\prime} \in S$, a vertex subset of some cluster which is most potent for $S^{\prime}$. Then, find the smallest vertex subsets of the clusters which have some signature in $S$ and add them to $V^{*}$ in decreasing order of the size of their cluster as long as $\left|V^{*}\right| \leq k$. Finally, add to $V^{*}$ arbitrary vertices until $\left|V^{*}\right|=k$. This algorithm finds an optimal solution because at least one of the possibilities checked above corresponds to an optimal solution and by the claims above.

It remains to show the running time: There are at most $2^{\ell}$ possibilities for $V^{*} \cap V_{\mathrm{VDS}}$. For each signature of a cluster $C_{i}$, of which there are at most $2^{\ell}$, there are at most $2^{2^{\ell}}$ possibilities for the set of vertex signatures of a subset of $C_{i}$. Hence, the signature of $V^{*}$ is the subset of a set of size $2^{\ell} \cdot 2^{2^{\ell}}$, meaning that there are at most $2^{2^{2^{O(\ell)}}}$ possibilities for the signature of $V^{*}$. Hence, the algorithm checks at most $2^{2^{2^{O(\ell)}}}$ possibilities. To see that the cluster vertex subsets added to $V^{*}$ for each possibility can be computed in polynomial time, observe that the it suffices to iterate over each cluster, find its signature and the signature of its vertices and accumulate the largest ones into a dictionary data structure indexed by the size of the clusters.

Directed Closeness Improvement. We now investigate the problem DireCTED CloseNESS IMPROVEMENT of improving the closeness centrality of a vertex $z$ on directed, unweighted graphs. Herein, the closeness centrality is measured by sum of the multiplicative inverse dis- 
tances from $z$ to the other vertice 3 . We show that the problem remains W[2]-hard with respect to the number $k$ of added arcs, even on directed acyclic graphs and even if the diameter of the graph is 4 . Analogously to the undirected variant, we show that we can maximize the closeness centrality of a vertex $z$ in a directed graph by adding $\operatorname{arcs}$ adjacent to $z$ :

Lemma 2. Let $I=(G=(V, E), z, k, r)$ be a Directed Closeness Improvement instance. If $I$ is a YES-instance, then there is a solution $S$ for $I$ where for each arc $a \in S$, the source vertex is $z$.

Proof. The proof is analogous to the one of Lemma 1; If an optimal solution $S$ contains an arc $a:=(u, v), u, v \neq z$, then any shortest path from $z$ to some vertex $w$ containing the arc $a$ becomes even shorter if $(u, v)$ is replaced by $(z, v)$. If $(z, v)$ already exists, then no shortest path from $z$ contains $a$; hence, it can be replaced by an arbitrary arc with source $z$. Furthermore, an $\operatorname{arc} a^{\prime}$ where $z$ is the endpoint does not improve the closeness centrality of $z$ at all, as any path from $z$ containing $a^{\prime}$ contains a loop and thus is no shortest path.

Lemma 2 directly implies that Directed Closeness Improvement is in XP with respect to the number of arc additions:

Corollary 3. Directed Closeness Improvement can be solved in $O\left(n^{k} \cdot(n+m)\right)$ time, where $k$ is the number of arc additions and thus is in XP with respect to the parameter number of arc additions.

Theorem 4. Directed Closeness Improvement is NP-hard and W[2]-hard with respect to the number $k$ of edge additions on directed acyclic graphs.

Proof. The proof uses a parameterized reduction from SET Coven with the parameter number of subsets $k$, which is be W[2]-hard [10]. Let $I=\left(\mathcal{F}=\left\{F_{1}, \ldots, F_{m}\right\}, U=\left\{s_{1}, \ldots, s_{n}\right\}, k\right)$ be a Set Cover instance. We reduce $I$ to a Closeness Improvement instance $I^{\prime}=(G=$ $\left.(V, A), z, k, k+\frac{n}{2}\right)$, where $G$ is a directed, unweighted graph constructed as follows: For each $s_{i} \in$ $U$ and each $F_{j} \in \mathcal{F}$, we add a vertex $v_{i}$ or $u_{j}$ to the graph, respectively. Furthermore, if $s_{i} \in F_{j}$ for any $s_{i} \in U, F_{j} \in \mathcal{F}$, then we add an $\operatorname{arc}\left(v_{j}, u_{i}\right)$. Finally, we add a vertex $z$ to the constructed graph. We provide an example in Fig. 3. It is easy to see that the constructed directed graph is acyclic.

Before showing the correctness of the reduction, we state and prove the following observation: The closeness centrality of $z$ can be maximally increased by adding arcs from $z$ to $v_{j}$.

First of all, the closeness centrality of $z$ can be maximally increased if the source of each added arc is $z$. Otherwise, if $z$ is the target of an arc, then we either introduced a loop, or the source of the arc remains unreachable from $z$. If $z$ is neither the source nor the target of the arc, then all introduced shortest paths containing this arc become even shorter if we replace the source of the $\operatorname{arc}$ by $z$. Finally, an $\operatorname{arc}\left(z, u_{i}\right)$ can be replaced by $\left(z, v_{j}\right)$, where $\left(v_{j}, u_{i}\right) \in E$. By adding the arc $\left(z, u_{i}\right)$, the distance from $z$ to $u_{i}$ is decreased to 1 . An $\operatorname{arc}\left(z, v_{j}\right)$ decreases the distance from $z$ to $v_{j}$ to 1 and the distance of at least one more vertex $u_{i}$ to 2 . Hence, by adding $\operatorname{arcs}$ from $z$ to $v_{j}$, we obtain a larger closeness centrality of $z$ compared to adding edges from $z$ to $u_{i}$.

We show that the reduction is correct, that is, $I$ is a Yes-instance if and only if $I^{\prime}$ is a YES-instance.

$\Rightarrow$ : If $I$ is a Yes-instance, then there is an $\mathcal{F}^{\prime} \subseteq \mathcal{F}$ of size $k$ such that $\bigcup_{F_{j} \in \mathcal{F}^{\prime}}=U$. By adding $k$ arcs $\left(z, v_{j}\right), F_{j} \in \mathcal{F}^{\prime}$, there are $k$ vertices with distance 1 from $z$, and each vertex

\footnotetext{
${ }^{3}$ It is easy to check that all our results also hold if the closeness centrality is measured by sum of the multiplicative inverse distances from the other vertices to $z$.
} 
in $\left\{u_{1}, \ldots, u_{n}\right\}$ has distance 2 from $z$. Hence, $c_{z}$ can be increased to $k+\frac{n}{2}$ and $I^{\prime}$ is a Yesinstance.

$\Leftarrow$ : If $I$ is not a YES-instance, then there is no such set $\mathcal{F}^{\prime} \subseteq \mathcal{F}$ of size $k$ such that $\cup_{F_{j} \in \mathcal{F}^{\prime}}=$ $U$.

After adding $k$ arcs from $z$ to vertices in $\left\{v_{1}, \ldots, v_{m}\right\}$, there is at least one vertex $u_{i}$ such that there is no path from $z$ to $u_{i}$. Summing up, $c_{z}$ can be increased to at most $k+\frac{n^{\prime}}{2}$ for $n^{\prime}<n$ and $I^{\prime}$ is a No-instance.

In the next theorem, we slightly modify the reduction in the proof of Theorem 4 in order to show that Directed Closeness Improvement remains W[2]-hard on directed graphs with diameter 4 .

Theorem 5. Directed Closeness Improvement is NP-hard and W[2]-hard with respect to the number $k$ of edge additions on directed graphs with diameter 4.

Proof. Let $I=\left(\mathcal{F}=\left\{F_{1}, \ldots, F_{m}\right\}, U=\left\{s_{1}, \ldots, s_{n}\right\}, k\right)$ be a SET Cover instance. We construct a Directed Closeness Improvement instance $I^{\prime}=(G=(V, E), z, k, r)$ as follows. First we construct a directed graph as described in the reduction in the proof of Theorem 4 . Then we add $m$ vertices $w_{i}$ and $2 m \operatorname{arcs}\left(z, w_{i}\right),\left(w_{i}, v_{i}\right)$ for each $1 \leq i \leq m$. Additionally, for each $u_{i}, v_{i}$ and $w_{i} \in V$, we add the $\operatorname{arcs}\left(u_{i}, z\right),\left(v_{i}, z\right)$ and $\left(w_{i}, z\right)$ to $G$. Finally, we set $r=k+2 n-\frac{k}{2}$.

The constructed graph is a directed graph with diameter 3: From $z$, the length of shortest paths to the other vertices is at most 3 . The distance from each vertex $w_{i}, v_{i}$ and $u_{i}$ to any vertex $u_{j}$ is at most 4 , and the distance from these vertices to any vertex $v_{j}$ is at most 3 . Hence, $G$ is a strongly connected directed graph with diameter 4 .

Analogously to the reduction in Theorem 4, there is an optimal solution for $I^{\prime}$ which only contains arcs where $z$ is the source and some of the vertices $v_{i}$ are the target - the proof for this statement is the same as the one in the referred theorem.

It remains to show that the reduction is correct, that is $I^{\prime}$ is a Yes-instance if and only if $I$ is a YeS-instance:

$\Rightarrow$ : If $I$ is a YES-instance, then there is an $\mathcal{F}^{\prime} \subseteq \mathcal{F}$ of size $k$ such that $\bigcup_{F_{j} \in \mathcal{F}^{\prime}}=U$. By adding $k$ arcs $\left(z, v_{j}\right), F_{j} \in \mathcal{F}^{\prime}$, there are $k$ vertices $v_{i}$ with distance 1 from $z$, and each vertex in $\left\{u_{1}, \ldots, u_{n}\right\}$ has distance 2 from $z$. Moreover, the other $n-k$ vertices $v_{i}$ have distance 2 from $z$, and each vertex $w_{i}$ has distance 1 from $z$. Hence, $c_{z}$ can be increased to $r=k+n+\frac{n+(n-k)}{2}$ and $I^{\prime}$ is a Yes-instance. $U$.

$\Leftarrow$ : If $I$ is not a Yes-instance, then there is no such set $\mathcal{F}^{\prime} \subseteq \mathcal{F}$ of size $k$ such that $\cup_{F_{j} \in \mathcal{F}^{\prime}}=$

After adding $k$ arcs from $z$ to vertices in $\left\{v_{1}, \ldots, v_{m}\right\}$, there is at least one vertex $u_{i}$ such that there is no path from $z$ to $u_{i}$. Hence, there are $n$ vertices $w_{i}$ and $k$ vertices $v_{i}$ with distance 1 from $z$ and $n-k$ vertices $u_{i}$ with distance 2 from $z$. Furthermore, there are $n^{\prime}<n$ vertices $u_{i}$ with distance 2 from $z$, and there is at least one vertex $u_{i}$ with distance 3 from $z$. Summing up, $c_{z}$ can be increased to at most $k+n+\frac{n^{\prime}+(n-k)}{2}+\frac{1}{3}$ for $n^{\prime}<n$ and $I^{\prime}$ is a No-instance.

The computational complexity of Directed Closeness Centrality on directed graphs with diameter 3 remains open. However, analogously to the problem variant with undirected input graphs, it is not hard to show that the problem is polynomial-time solvable on graphs with diameter at most 2 . 


\section{Betweenness Centrality}

We now investigate the problem of increasing the betweenness centrality of a vertex in a graph by inserting a certain number of edges into the graph. We remark that the betweenness centrality of a vertex in an undirected graph can be computed in $O(n \cdot m)$ time [4]. We show that, similar to Closeness Improvement, Betweenness Improvement is W[2]-hard with respect to the parameter number of edge additions and FPT with respect to the combination of the number of edge additions and the distance to a cluster graph.

First, we make an important observation that will help us in our proofs. Analogous to Lemma 1, we show that to improve the betweenness of a vertex by adding edges, it always makes sense to add only edges adjacent to that vertex.

Lemma 3. Let $I=(G, z, k, r)$ be a Betweenness Improvement instance. If $I$ is a Yesinstance, then there is an optimal solution that only contains edges where one endpoint is $z$.

Proof. Let $S$ be a solution for $I$, and let $e:=\left\{u_{i}, u_{j}\right\} \in S$. Furthermore, assume that $e$ introduces at least one shortest path containing $z$ (if it does not, then $e$ can be replaced by any edge containing $z$ ). Without loss of generality, assume $u_{i}$ precedes $u_{j}$ on each of these paths. Then by replacing $e$ by $e^{\prime}:=\left\{z, u_{j}\right\}$ in $S$, the distance between $z$ and $u_{j}$ decreases to 1 and the shortest paths previously containing $e$ now contain $e^{\prime}$. Hence, $b_{z}$ does not decrease.

Hence, if we compute a solution for some BetweEnNEss Improvement instance, we need to find a subset of the graph's vertices of size $k$ such that adding an edge between $z$ and these vertices maximally increases the betweenness centrality of $z$. This directly implies the following corollary:

Corollary 4. Betweenness Improvement is solvable in $O\left(n^{k} \cdot(n+m)\right)$ time where $k$ is the number of edge additions and thus is in XP with respect to the parameter number of edge additions.

Hardness Results. We show that Betweenness Improvement is W[2]-hard with respect to the parameter number of edge additions by a parameterized reduction from Dominating SET on graphs with diameter 3. Furthermore, we show that the problem is NP-hard on graphs with diameter 3 and H-index 4.

Theorem 6. Betweenness ImProvement is NP-hard and W[2]-hard with respect to the parameter number $k$ of edge additions on graphs with diameter 3. Moreover, unless the Exponential Time Hypothesis fails, Betweenness Improvement does not allow an algorithm with running time $f(k) \cdot n^{o(k)}$.

Proof. We give a parameterized reduction from Dominating Set, which also directly implies the running time lower bound when assuming ETH [8]. Let $I=(G=(U, E), k)$ be a DomINAting Set instance, where $U=\left\{u_{1}, \ldots, u_{n}\right\}$. We construct a Betweenness Improvement instance

$$
I^{\prime}=\left(G^{\prime}=\left(V, E^{\prime}\right), z_{1}, k, r=\alpha k+\frac{2}{3} \alpha(n-k)+\frac{1}{2}\left(k+\alpha+\left(\begin{array}{l}
\alpha \\
2
\end{array}\right)\right)\right),
$$

where $\alpha>\frac{3 k(k-1)}{2}$. The graph $G^{\prime}$ is constructed as follows. For each $u_{i} \in U$, we add a vertex $u_{i}^{\prime}$ to $G^{\prime}$. Also, for each edge $\left\{u_{i}, u_{j}\right\} \in E$, we add an edge $\left\{u_{i}^{\prime}, u_{j}^{\prime}\right\}$ to $E^{\prime}$. We set $U^{\prime}:=\left\{u_{1}^{\prime}, \ldots, u_{n}^{\prime}\right\}$. Next, we add the vertices $\left\{z_{1}, z_{3}, z_{4}\right\}$ and $Z_{2}=\left\{z_{2_{1}}, \ldots, z_{2_{\alpha}}\right\}$ to $G^{\prime}$. For each $z_{2_{i}} \in Z_{2}$, we add two edges $\left\{z_{1}, z_{2_{i}}\right\}$ and $\left\{z_{2_{i}}, z_{3}\right\}$ to $G^{\prime}$. Furthermore, we add the edges $\left\{z_{1}, z_{3}\right\},\left\{z_{1}, z_{4}\right\}$ 

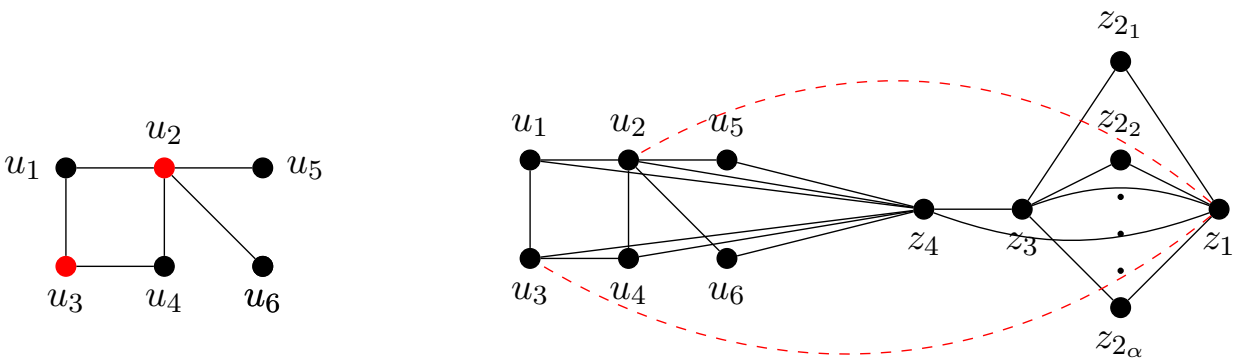

Figure 4: Parameterized reduction from Dominating Set to Betweenness Improvement. Left: A Dominating Set instance $(I=(G, k=2))$. The red colored vertices $u_{2}, u_{3}$ form a solution. Right: The constructed Betweenness Improvement instance $I^{\prime}=\left(G, z_{1}, k, r\right)$. The red, dashed edges form a solution.

and $\left\{z_{3}, z_{4}\right\}$. Finally, for each vertex $u_{i}^{\prime} \in U^{\prime}$, we add an edge $\left\{z_{4}, u_{i}^{\prime}\right\}$. Fig. 4 illustrates the construction. It is easy to check that $G^{\prime}$ has diameter 3 .

As $z_{1}$ is adjacent to all vertices except the ones in $U^{\prime}$, a solution $S$ for $I^{\prime}$ contains only edges where one endpoint is $z_{1}$ and each other one is in $U^{\prime}$ (Lemma 3).

We now show that $I^{\prime}$ is a YES-instance if and only if $I$ is a YES-instance: First, if $I$ is a No-instance, we show that there is an upper bound $r_{u}<r$ such that $b_{z_{1}}$ can be increased to at most $r_{u}$ by adding at most $k$ edges to $G^{\prime}$. Second, if $I$ is a Yes-instance, we provide a lower bound $r_{\ell} \geq r$ such that $b_{z_{1}}$ can be increased to at least $r_{\ell}$ by adding at most $k$ edges to $G^{\prime}$. Both $r_{\ell}$ and $r_{u}$ depend on $\alpha$, which determines the size of $G^{\prime}$. Finally, we determine a minimum value for $\alpha$ such that $r_{\ell}$ and $r_{u}$ are strict bounds.

$\Rightarrow$ The input graph contains a dominating set $U_{D S} \subseteq U$ of size $k$. We say that $U_{D S}^{\prime}$ is the set of vertices in the constructed graph which correspond to the vertices in $U_{D S}$. Then, by adding $k$ edges between $z_{1}$ and the vertices in $U_{D S}^{\prime}$, for the following pairs of vertices there are shortest paths containing $z_{1}$ :

- For each pair $\left(u^{\prime} \in U_{D S}^{\prime}, z \in Z_{2}\right)$, there is one shortest path of length 2 , containing $z_{1}$. The number of such pairs is $\alpha k$.

- For each pair $\left(u^{\prime} \in U^{\prime} \backslash U_{D S}^{\prime}, z \in Z_{2}\right)$, two out of three shortest paths of length 3 between $u^{\prime}$ and the vertices in $z$ contain $z_{1}$ : One contains $z_{1}$ and a member of the dominating set, one contains $z_{1}$ and $z_{4}$, and one contains $z_{3}$ and $z_{4}$. The number of such pairs is $\alpha(n-k)$.

- For each pair $\left(u^{\prime} \in U_{D S}^{\prime}, z_{3}\right)$, there are two shortest paths of length 2 between $u^{\prime}$ and $z_{3}$ : One contains $z_{1}$, the other one contains $z_{4}$. The number of such pairs is $k$.

- For each pair $\left(z_{2_{i}}, z_{2_{j}} \in Z_{2} \mid i \neq j\right)$, there are two shortest paths of length 2 between $z_{2_{i}}$ and $z_{2_{j}}$ : One contains $z_{1}$, the other one contains $z_{3}$. The number of such pairs is $\left(\begin{array}{l}\alpha \\ 2\end{array}\right)$.

- For each pair $\left(z_{2_{i}} \in Z_{2}, z_{4}\right)$, there are two shortest paths of length 2: One contains $z_{3}$ and the other one contains $z_{1}$. The number of such pairs is $\alpha$

In total,

$$
b_{z_{1}} \geq \alpha k+\frac{2 \alpha(n-k)}{3}+\frac{k}{2}+\frac{\left(\begin{array}{l}
\alpha \\
2
\end{array}\right)}{2}+\frac{\alpha}{2},
$$

which can be simplified to

$$
b_{z_{1}} \geq \alpha k+\frac{2 \alpha(n-k)}{3}+\frac{k+\alpha+\left(\begin{array}{c}
\alpha \\
2
\end{array}\right)}{2}=: r_{\ell}
$$

$\Leftarrow$ We prove the reverse direction by contraposition. That is, we show that $I^{\prime}$ is a Noinstance if $I$ is a No-instance. If the input instance does not admit a dominating set of size at 
most $k$, then there is at least one vertex which cannot be dominated. We analyze the number of shortest paths in the constructed BETWEENNESS IMPROVEMENT instance after adding $k$ edges between $z_{1}$ and vertices in $U^{\prime}$. We set $U^{\prime \prime} \subseteq U^{\prime}:=\left\{u_{i}^{\prime} \in U^{\prime} \mid\left\{z_{1}, u_{i}^{\prime}\right\} \in S\right\}$. Furthermore, let $\ell$ be the number of vertices that are undominated in $G^{\prime}$ after adding the edges in $S$, i.e. which are not adjacent to $z_{1}$ and which do not have a neighbor adjacent to $z_{1}$. As $G$ does not admit a dominating set of size $k$, it holds that $\ell \geq 1$.

- For each pair $\left(u^{\prime} \in U^{\prime \prime}, z \in Z_{2}\right.$ ), there is one shortest path of length 2 , containing $z_{1}$. The number of such pairs is $\alpha k$.

- For each pair $\left(u^{\prime} \in U^{\prime} \backslash U^{\prime \prime}, z \in Z_{2}\right)$ where $u^{\prime}$ is a neighbor of one of the vertices in $U^{\prime \prime}$, two out of three shortest paths of length 3 between $u^{\prime}$ and the vertices in $z$ contain $z_{1}$ : One contains $z_{1}$ and a member of the dominating set, one contains $z_{1}$ and $z_{4}$, and one contains $z_{3}$ and $z_{4}$. The number of such pairs is $\alpha(n-k-\ell)$.

- For each pair $\left(u_{i}^{\prime}, u_{j}^{\prime} \in U^{\prime}\right)$, there is a path of length 2 containing $z_{4}$. If the vertices in $U^{\prime}$ are not adjacent, then this is the shortest path. Additionally, there may be another shortest path containing $z_{1}$ of length 2 , introduced by the edges in $S$. Hence, for each of up to $\left(\begin{array}{l}k \\ 2\end{array}\right)$ pairs of vertices, one out of two shortest path contain $z_{1}$.

- For each pair $\left(u^{\prime} \in U^{\prime} \backslash U^{\prime \prime}, z \in Z_{2}\right)$ where $u^{\prime}$ is not a neighbor of one of the vertices in $U^{\prime \prime}$, there are two shortest paths betweens $u^{\prime}$ and $z$ of length 3 : One contains $z_{1}$ and $z_{4}$, the other one contains $z_{3}$ and $z_{4}$. The number of such pairs is $\alpha \ell$.

- For each pair $\left(u^{\prime} \in U^{\prime \prime}, z_{3}\right)$, there are two shortest paths of length 2 between $u^{\prime}$ and $z_{3}$ : One contains $z_{1}$, the other one contains $z_{4}$. The number of such pairs is $k$.

- For each pair $\left(z_{2_{i}}, z_{2_{j}} \in Z_{2} \mid i \neq j\right)$, there are two shortest paths of length 2 between $z_{2_{i}}$ and $z_{2_{j}}$ : One contains $z_{1}$, the other one contains $z_{3}$. The number of such pairs is $\left(\begin{array}{l}\alpha \\ 2\end{array}\right)$.

- For each pair $\left(z_{2_{i}} \in Z_{2}, z_{4}\right)$, there are two shortest paths of length 2: One contains $z_{3}$ and the other one contains $z_{1}$. The number of such pairs is $\alpha$.

In total,

$$
b_{z_{1}} \leq \alpha k+\frac{2 \alpha(n-k-\ell)}{3}+\frac{\left(\begin{array}{l}
k \\
2
\end{array}\right)}{2}+\frac{\alpha \ell}{2}+\frac{k}{2}+\frac{\left(\begin{array}{l}
\alpha \\
2
\end{array}\right)}{2}+\frac{\alpha}{2},
$$

which can be simplified to

$$
b_{z_{1}} \leq \alpha k+\frac{2 \alpha(n-k-\ell)}{3}+\frac{\left(\begin{array}{l}
k \\
2
\end{array}\right)+\alpha(\ell+1)+k+\left(\begin{array}{l}
\alpha \\
2
\end{array}\right)}{2}=: r_{u} .
$$

In the last step, we need to determine a proper value for $\alpha$ such that $r_{u}<r_{\ell}$. Hence, the inequality that needs to be satisfied is

$$
\alpha k+\frac{2 \alpha(n-k-\ell)}{3}+\frac{\left(\begin{array}{l}
k \\
2
\end{array}\right)+\alpha(\ell+1)+k+\left(\begin{array}{l}
\alpha \\
2
\end{array}\right)}{2}<\alpha k+\frac{2 \alpha(n-k)}{3}+\frac{k+\alpha+\left(\begin{array}{l}
\alpha \\
2
\end{array}\right)}{2}
$$

for each $n, k, \ell \in \mathbb{N}, k \leq n, 1 \leq \ell \leq n$. This equation can be transformed to

$$
\frac{\alpha \ell}{3}>\left(\begin{array}{l}
k \\
2
\end{array}\right)
$$

By setting $\ell=1$ and transforming the binomial coefficient, we get

$$
\alpha>\frac{3 k(k-1)}{2} .
$$

Hence, by setting $\alpha$ to a value strictly larger than $\frac{3 k(k-1)}{2}$, the reduction is correct. Furthermore, the reduction is computable in fpt time: As the size of $I^{\prime}$ is polynomial to the size of $I, G^{\prime}$ can be constructed even in polynomial time. 
By closer inspection of the reduction, we can also show that BetweEnNESs IMPRovement remains hard on graphs with diameter 3 and H-index 4.

Corollary 5. Betweenness ImProvement is NP-hard on graphs with diameter 3 and $H$-index 4.

Proof. Let $I=(G, k)$ be a Dominating Set instance, where $G$ is a graph with maximum degree three. Let $G^{\prime}$ be the graph constructed by the reduction used in the proof of Theorem 6 . Then each vertex except $z_{1}, z_{3}$, and $z_{4}$ has degree at most four. Hence, the h-index of $G^{\prime}$ is at most four. As Dominating SET is NP-hard even on planar graphs with degree three [14], BetweEnness ImPRovement remains NP-hard on graphs with h-index four. Furthermore, the constructed graph has diameter 3 .

Algorithmic Result. We also derive a positive result for Betweenness Improvement. We show that the problem is fixed-parameter tractable with respect to the combined parameter distance to cluster and number of edge additions.

Theorem 7. Betweenness Improvement is solvable in time $2^{O\left(2^{2^{\ell}} \cdot k \log k\right)} \cdot n^{O(1)}$, where $\ell$ is the distance of $G$ to a cluster graph, and thus is in FPT with respect to the combined parame$\operatorname{ter}(k, \ell)$.

Proof. Let $(G, z, k, r)$ be a Betweenness Improvement instance, where the set $V_{\text {VDS }} \subset V$ is a cluster vertex deletion set of size $\ell$, that is, $G\left[V \backslash V_{\mathrm{VDS}}\right]$ is a cluster graph with connected components (clusters) $\left\{C_{1}, \ldots, C_{s}\right\}=: C$. Since a cluster vertex deletion set of size $\ell$ can be found in $O\left(1.92^{\ell} \cdot(n+m)\right)$ time if it exists [3, 15], we may assume that $V_{\mathrm{VDS}}$ is given. The basic idea is similar to Theorem 3. First, we determine the intersection of an optimal solution with $V_{\text {VDS }}$. To find the vertices in $V \backslash V_{\text {VDS }}$ we assign signatures to clusters and vertices in clusters based on their neighborhood in $V_{V D S}$. We then find the signatures in an optimal solution and the optimal vertices for each signature. A difference to Theorem 3 is that, once we have determined the signatures of vertices in an optimal solution, it still matters how many vertices we take for each signature.

Let $V^{*}$ be the set of endpoints different from $z$ of the edges in an optimal solution. By Lemma 3 we may assume that $\left|V^{*}\right|=k$. The first step in the algorithm is to iterate over all $2^{\ell}$ possibilities for putting $V^{*} \cap V_{V D S}$ in the output solution. Assume henceforth that we are in the iteration in which we have found $V^{*} \cap V_{\text {VDS }}$.

Define for each cluster $C_{i}$ its cluster signature as the set of neighbors of $C_{i}$ in $V_{\mathrm{VDS}} \cup\{z\}$. From $V^{*}$ we get a subset $S$ of the set of all $2^{\ell}$ possible cluster signatures by putting into $S$ all signatures of clusters which have nonempty intersection with $V^{*}$. That is, $\mathcal{S}=\left\{N\left(C_{i}\right) \cap\left(V_{\mathrm{VDS}} \cup\{z\}\right) \mid\right.$ $i \in\{1, \ldots, s\}\}$, where $N\left(C_{i}\right)=\bigcup_{v \in C_{i}} N(v)$. The second step in the algorithm is to iterate over all $2^{2^{\ell}}$ possibilities for $\mathcal{S}$. Assume below that we are in the iteration in which we have found $\mathcal{S}$.

Define for each vertex $v \in V \backslash\left(V_{\mathrm{VDS}} \cup\{z\}\right)$ its vertex signature as the set $N(v) \cap\left(V_{\mathrm{VDS}} \cup\{z\}\right)$. From $V^{*}$, for each cluster signature $S \in \mathcal{S}$, we obtain a family $T_{S}$ of sets of vertex signatures by, for each cluster $C_{i}$ with signature $S$ that has nonempty intersection with $V^{*}$, putting into $T_{S}$ the set $\left\{N(v) \cap V_{\text {VDS }} \mid v \in C_{i} \cap V_{\text {VDS }}\right\}$. The third step in the algorithm is to iterate for each $S \in \mathcal{S}$ over all $2^{2^{\ell}}$ possible families $T_{S}$. In total, these are at most $2^{\ell} \cdot 2^{2^{2^{\ell}}}$ possibilities. Assume henceforth that we are in the iteration in which we have found $T_{S}$ for each $S \in \mathcal{S}$.

As fourth step in the algorithm we find for each $S \in \mathcal{S}$ and each $S^{\prime} \in T_{S}$ the number $n_{S, S^{\prime}}$ of clusters $C_{i}$ such that $C_{i} \cap V^{*} \neq \emptyset, C_{i}$ has signature $S^{\prime}$, and the set of vertex signatures of vertices in $C_{i} \cap V^{*}$ is exactly $S^{\prime}$. We do this by iterating over all at most $\left(2^{\ell} \cdot 2^{2^{\ell}}\right)^{k}$ possibilities. 
Assume henceforth that we are in the iteration in which we have found $n_{S, S^{\prime}}$ for each $S \in \mathcal{S}$ and each $S^{\prime} \in T_{S}$.

As a fifth step in the algorithm we find for each of the $n_{S, S^{\prime}}$ clusters $C_{i}$ as above, for each vertex signature in $s \in S^{\prime}$ the number $n_{S, S^{\prime}, s}$ of vertices in $C_{i} \cap V^{*}$ with signature $s$. Again, we iterate over all at most $\left(2^{\ell} \cdot 2^{2^{\ell}} \cdot 2^{\ell} \cdot k\right)^{k}$ possibilities. Assume henceforth that we are in the iteration in which we have found $n_{S, S^{\prime}, s}$.

Say that a cluster $C_{i}$ is eligible for $S, S^{\prime}$ if it has cluster signature $S$ and for each vertex signature $s \in S^{\prime}$ there are $n_{S, S^{\prime}, s}$ vertices with signature $s$ in $C_{i}$. We now claim that, without loss of generality, among clusters that are eligible for $S, S^{\prime}$, set $V^{*}$ contains only vertices from the $k$ largest such clusters. Assume otherwise. Hence, there is a cluster $C_{i}$ among the $k$ largest clusters eligible for $S, S^{\prime}$ and a cluster $C_{j}$ which is eligible for $S, S^{\prime}$ but not among the $k$ largest such clusters. (Recall that we are in an iteration in which we have found $S, S^{\prime}, n_{S, S^{\prime}}$, and $n_{S, S^{\prime}, s}$ as defined and hence, $C_{j}$ exists.) Obtain $W^{*}$ from $V^{*}$ by replacing each vertex in $C_{j} \cap V^{*}$ with a vertex in $C_{i}$ with the same signature; call the vertices in $C_{i} \cap W^{*}$ the replacements of the vertices in $V^{*}$. The betweenness centrality of $z$ with respect to $W^{*}$ is at least the one with respect to $V^{*}$. Indeed, each shortest path with respect to $V^{*}$ that contains $z$ and some vertices in $C_{j} \cap V^{*}$ induces a shortest path with respect to $W^{*}$ containing $z$ and the corresponding replacements in $C_{i} \cap W^{*}$. Thus, the claim holds.

The sixth and final step in the algorithm is thus to try all possibilities to mark $n_{S, S^{\prime}}$ clusters which are eligible for $S, S^{\prime}$ and to put, for each marked cluster and each $s \in S^{\prime}$ a set of $n_{S, S^{\prime}, s}$ arbitrary vertices of signature $s$ in the marked cluster into the output solution. There are at most $\left(2^{\ell} \cdot 2^{2^{\ell}} \cdot k^{2}\right)^{k}$ possibilities. By the claim and since we can replace vertices with the same signatures in the marked clusters in $V^{*}$, in one of the tried possibilities, we will find an optimal solution.

Directed Betweenness Improvement. We now cover results for the problem of improving the betweenness centrality of a vertex in a directed, unweighted graphs. First, we define betweenness centrality for directed, unweighted graphs, as the definition due to Freeman 12 ] only measures the centrality over all unordered subsets of vertices of size two. A very natural definition, which is equivalent to the one used in further literature (e.g. by White and Borgatti [25]) is to measure the ratio of shortest paths containing a certain vertex $z$ for both orders of any pair of vertices: $b_{z}=\sum_{s \in V} \sum \frac{\sigma_{s t z}}{\sigma_{s t}}$. Herein $s, t \neq z$ and the second sum is taken over all $t \in V$ such that $t \neq s$ and $\sigma_{s t} \neq 0$. Using this definition, Directed Betweenness Improvement is defined analogously to BetweEnness Improvement.

Analogously to the undirected problem variant, we show that we can maximize the betweenness centrality of a vertex $z$ by adding arcs incident to $z$.

Lemma 4. If a Directed Betweenness Improvement instance $I=(G=(V, A), z, k, r)$ is a YES-instance, then there is a solution $S$ that only contains arcs where either the source or the target is $z$.

Proof. Assume $S$ contains an $\operatorname{arc}\left(u_{1}, u_{2}\right)$ such that $u_{1} \neq z$ and $u_{2} \neq z$. Let $v_{1}, v_{2} \in V$ such that $\left(u_{1}, u_{2}\right)$ introduced a shortest path from $v_{1}$ to $v_{2}$ containing $z$ and the $\operatorname{arc}\left(u_{1}, u_{2}\right)$. It is clear that $u_{2}$ must have been connected to $z$ by a path before adding the arc $\left(u_{1}, u_{2}\right)$. Furthermore, it is clear $\sigma_{v_{1} v_{2} z} \leq 1$, as there max be other paths from $v_{1}$ to $v_{2}$ not containing $z$.

However, the shortest paths introduced by $\left(u_{1}, u_{2}\right)$ necessarily contain $u_{1}$ and $u_{2}$; hence, these paths can be contracted by replacing $\left(u_{1}, u_{2}\right)$ by $\left(u_{1}, z\right)$. By this, we do not decrease $b_{z}$ : Let $\ell$ be the length of a shortest path from $v_{1}$ to $v_{2}$ which contains $\left(u_{1}, u_{2}\right)$ and $z$. Then, after replacing $\left(u_{1}, u_{2}\right)$ by $\left(u_{1}, z\right)$, there is exactly one shortest path of length $\ell^{\prime}<\ell$ from $v_{1}$ to $v_{2}$. Hence, $\sigma_{s t z}=1$. 

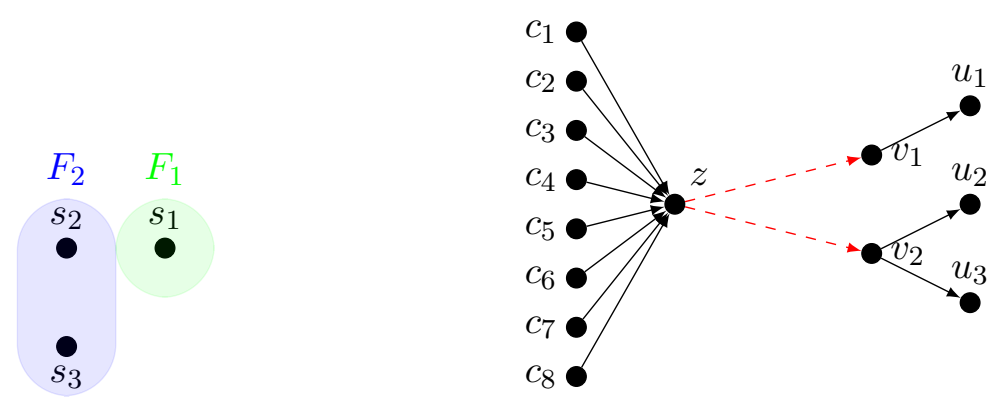

Figure 5: Parameterized reduction from Set Cover to Directed Betweenness ImproveMent. Left: A Set Cover instance $I=(U, \mathcal{F}, k=2)$ with solution $\left\{F_{1}, F_{2}\right\}$. Right: The constructed Directed Betweenness Improvement instance $I^{\prime}=\left(G, z, k=2, r=4 \frac{5}{6}\right)$. The red dashed edges imply a solution for $I^{\prime}$.

However, note that a solution $S$ for a Yes-instance $I=(G, z, k, r)$ may also contain arcs where $z$ is the source. For instance, $\left(G=\left\{z, v_{1}, v_{2}\right\}, A=\left\{\left(v_{1}, z\right)\right\}, z, 1,1\right)$ is a Yes-instance with solution $S=\left\{\left(z, v_{2}\right)\right\}$.

Corollary 6. Directed Betweenness Improvement is solvable in $O\left((2 n)^{k} \cdot(n+m)\right)$ time where $k$ is the number of edge additions, and thus is in XP with respect to the parameter number of edge additions.

Substantial improvement of this running time is unlikely, as Theorem 8 shows.

Theorem 8. Directed Betweenness Improvement is NP-hard and W[2]-hard with respect to the parameter number of arc additions $k$ on directed acyclic graphs.

Proof. We show a parameterized reduction from Set Cover. Let $I=\left(\mathcal{F}=\left\{F_{1}, \ldots, F_{m}\right\}, U=\right.$ $\left.\left\{s_{1}, \ldots, s_{n}\right\}\right)$ be a Set Cover instance. We construct a Directed Betweenness ImproveMENT instance $\left.I^{\prime}=(G=(V, A), z, k, k(1+n)+n)\right)$, where $G$ is a directed, unweighted graph. The construction is as follows:

- For each $s_{i} \in U$, add a vertex $u_{i}$. Set $V_{U}:=\left\{u_{1}, \ldots, u_{n}\right\}$.

- For each $F_{j} \in \mathcal{F}$, add a vertex $v_{j}$. Set $V_{V}:=\left\{v_{1}, \ldots, v_{m}\right\}$.

- Add the vertex $z$.

- Add the vertices $c_{1}, \ldots, c_{m(m+n-1)}$; the set of these vertices is denoted as $V_{C}$.

- For each $c \in V_{C}$, add the $\operatorname{arcs}(c, z)$.

- For each $u_{i} \in V_{U}$ and each $v_{j} \in V_{V}$, add an $\operatorname{arc}\left(v_{j}, u_{i}\right)$ if $s_{i} \in F_{J}$.

In Fig. 5, the reduction is illustrated. It is easy to see that the constructed directed graph is acyclic.

Let $I$ be a YES-instance and $S$ be an arc set of size at most $k$, such that $b_{z} \geq r$ in $G^{\prime}=$ $(V, A \cup S)$. We now show that for each $\operatorname{arc} a \in S$, the source is $z$ and the target is one of the vertices in $V_{V}$. First, from Lemma 4 we know that there is a solution $S^{\prime}$ of the same size where $z$ is an endpoint of each arc $a \in S^{\prime}$. Hence, in the following we assume that for each $a \in S$, one of its endpoints is $z$.

Moreover, if a solution $S$ contains an $\operatorname{arc}\left(z, u_{i}\right), u_{i} \in V_{U}$, we can replace it by an $\operatorname{arc}\left(z, v_{i}\right), v_{i} \in$ $V_{V}$ such that $s_{i} \in F_{j}$ without decreasing $b_{z}$ : The $\operatorname{arc}\left(z, u_{i}\right)$ introduces paths from the vertices $z$ and all its predecessors to $u_{i}$. By replacing $\left(z, u_{i}\right)$ by $\left(z, v_{j}\right)$, the paths remain, but additionally paths from $z$ and its predecessors to $v_{j}$ are added. Hence, $b_{z}$ does not decrease.

Furthermore, by adding the vertex set $V_{C}$ of size $m(m+n-1)$, we ensure that by adding $\operatorname{arcs}$ where the source is $z$, we obtain more (shortest) paths containing $z$ than by adding $\operatorname{arcs}$ 
where the endpoint is $z$ : Each arc from $z$ to a vertex in $V_{V}$ introduces at least $m(m+n-1)$ shortest paths containing $z$. However, adding an arc from a vertex in $V_{U}$ to $z$ introduces at most $m((m-1)+(n-1))$ paths containing $z$ : Each vertex in $V_{U}$ has at most $m$ predecessors in $V_{V}$. Furthermore, $z$ may have at most $m$ successors in $V_{V}$ and at most $(n-1)$ successors in $V_{U}$. Hence, by adding an arc from a vertex in $V_{U}$ to $z, c_{z}$ is increased by at most $m((m-1)+(n-1))$.

We now show that the reduction is correct, i.e. that $I$ is a Yes-instance if and only if $I^{\prime}$ is a YES-instance.

$\Rightarrow$ : If $I$ is a YES-instance, then there is a $\mathcal{F}^{\prime} \subseteq \mathcal{F}$ of size $k$ such that $\cup_{F_{j} \in \mathcal{F}^{\prime}}=U$. By adding $\operatorname{arcs}\left(z, v_{j}\right)$ for each $F_{j} \in \mathcal{F}^{\prime}$, the following shortest paths contain $z$ :

- For each $v_{j}$ such that $F_{j} \in \mathcal{F}^{\prime}$ and each $c \in V_{C}$, there is a shortest path from $c$ to $v_{j}$ containing $z$. As $\left|\mathcal{F}^{\prime}\right|=k$, the number of such shortest paths is $k(m(m+n-1))$.

- For each $u_{i}$ and each $c \in V_{C}$, there is a shortest path from $c$ to $u_{i}$ containing $z$. In total, the number of such paths is $n(m(m+n-1))$.

Hence, $b_{z}$ can be increased to $(k+n)(m(m+n-1))$ and $I^{\prime}$ is a YES-instance.

$\Leftarrow$ : If $I$ is not a Yes-instance, then there is no such set $\mathcal{F}^{\prime} \subseteq \mathcal{F}$ of size $k$ such that $\sum_{\mathcal{F}^{\prime}}=U$. Let $S$ be a set of size $k$ which contains arcs from vertices $v_{j}$ to $z$. The graph $G^{\prime}=(V, A \cup S)$ contains the following shortest paths, each containing $z$ :

- For each $v \in V_{V}$ which is the endpoint in an arc in $S$, and each $c \in V_{C}$, there is a shortest path from $c$ to $v$ containing $z$. As the target of all $\operatorname{arcs}$ in $S$ is a vertex in $V_{V}$ and $|S|=k$, the number of such shortest paths is $k(m(m+n-1))$.

- As $I$ is a No-instance, there is at least one vertex $u$ in $G^{\prime}$ such that there is no path from the vertices in $V_{C}$ to $u$. Hence, the number of paths from vertices in $V_{C}$ to vertices in $V_{U}$ is at most $n-1(m(m+n-1))$.

Hence, $b_{z}$ can be increased to at most $(k+n-1)(m(m+n-1))$ and $I^{\prime}$ is a YeS-instance.

\section{Conclusion and Outlook}

We studied the (parameterized) complexity of Closeness Improvement and Betweenness IMPROVEMENT with respect to the number $k$ of added edges and (unfortunately) obtained mostly hardness results even in several special cases that are relevant to practice. On the plus side, we obtained tractability results relating to the vertex-deletion distance to cluster graphs.

Our tractability results yield running times that are impractical and need to be improved. Some further questions that we left open are as follows. First, it is not hard to show that Closeness Improvement polynomial-time solvable on graphs of diameter 2. Is this also true for diameter 3? As we showed, for diameter 4 it is NP-hard. Noticeable is also that the problem seems to be harder on disconnected graphs. In particular, our reductions also imply NP-hardness for disconnected graphs where every connected component has diameter 2 .

There seem to be similarities between Dominating Set and Closeness Improvement, as indicated by our hardness reductions. Dominating SeT is fixed-parameter tractable with respect to the combined parameter maximum degree and $k$. Does the same hold for CLosenEsS Improvement? Similar questions extend to Betweenness Improvement. For BetweenNESS IMPROVEMENT it would also be interesting to see, whether in our fixed-parameter algorithm for the combined parameter solution size $k$ and the distance to cluster graph, we can remove the dependency on $k$. 


\section{References}

[1] A. M. Ambalath, R. Balasundaram, C. R. H, V. Koppula, N. Misra, G. Philip, and M. S. Ramanujan. On the Kernelization Complexity of Colorful Motifs. In Proceedings of the 5th International Symposium on Parameterized and Exact Computation (IPEC 2010), Lecture Notes in Computer Science, pages 14-25. Springer, 2010. 6]

[2] A. A. Bertossi. Dominating sets for split and bipartite graphs. Information Processing Letters, 19(1):37-40, 1984. 5

[3] A. Boral, M. Cygan, T. Kociumaka, and M. Pilipczuk. A Fast Branching Algorithm for Cluster Vertex Deletion. Theory of Computing Systems, 58(2):357-376, 2016. 6, 14

[4] U. Brandes. A faster algorithm for betweenness centrality. Journal of Mathematical Sociology, 25(2):163-177, 2001. 11

[5] U. Brandes. On variants of shortest-path betweenness centrality and their generic computation. Social Networks, 30(2):136-145, 2008. 2

[6] P. Crescenzi, G. D'angelo, L. Severini, and Y. Velaj. Greedily improving our own closeness centrality in a network. ACM Transactions on Knowledge Discovery from Data, 11(1):9, 2016. 2

[7] P. Csermely, A. London, L.-Y. Wu, and B. Uzzi. Structure and dynamics of core/periphery networks. Journal of Complex Networks, 1(2):93-123, 2013. 2

[8] M. Cygan, F. V. Fomin, Ł. Kowalik, D. Lokshtanov, D. Marx, M. Pilipczuk, M. Pilipczuk, and S. Saurabh. Parameterized Algorithms. Springer, 2015. 3, 4, 11

[9] G. D'Angelo, L. Severini, and Y. Velaj. On the maximum betweenness improvement problem. Electronic Notes in Theoretical Computer Science, 322:153-168, 2016. 2

[10] R. G. Downey and M. R. Fellows. Parameterized complexity. Springer, 2012. 5, 9

[11] D. Eppstein and E. S. Spiro. The h-Index of a Graph and its Application to Dynamic Subgraph Statistics. Journal of Graph Algorithms and Applications, 16(2):543-567, 2012. 3, 5

[12] L. C. Freeman. A set of measures of centrality based on betweenness. Sociometry, 40: $35-41,1977$. 15]

[13] L. C. Freeman. Centrality in social networks conceptual clarification. Social Networks, 1 (3):215-239, 1978. 1, 2

[14] M. R. Garey and D. S. Johnson. Computers and Intractability; A Guide to the Theory of NP-Completeness. W. H. Freeman \& Co., 1990. 5, 14

[15] F. Hüffner, C. Komusiewicz, H. Moser, and R. Niedermeier. Fixed-parameter algorithms for cluster vertex deletion. Theory of Computing Systems, 47(1):196-217, 2010. 6, 14

[16] R. Impagliazzo and R. Paturi. Complexity of k-SAT. In Proceeding of the 14 th Annual IEEE Conference on Computational Complexity (CCC '99), pages 237-240, 1999. 3

[17] R. Impagliazzo, R. Paturi, and F. Zane. Which problems have strongly exponential complexity? In Proceedings 39th Annual Symposium on Foundations of Computer Science (FOCS '98), pages 653-662, 1998. 3 
[18] D. Lokshtanov, N. Misra, G. Philip, M. S. Ramanujan, and S. Saurabh. Hardness of rdominating set on graphs of diameter $(\mathrm{r}+1)$. In Proceedings of the 8th International Symposium on Parameterized and Exact Computation (IPEC 2013), pages 255-267, 2013. 5, 6 .

[19] M. Newman. Networks: An Introduction. Oxford University Press, 2010. 1, 2

[20] M. E. Newman. A measure of betweenness centrality based on random walks. Social networks, 27(1):39-54, 2005. 2

[21] K. Okamoto, W. Chen, and X.-Y. Li. Ranking of closeness centrality for large-scale social networks. In International Workshop on Frontiers in Algorithmics, pages 186-195. Springer, 2008. 2

[22] T. Opsahl, F. Agneessens, and J. Skvoretz. Node centrality in weighted networks: Generalizing degree and shortest paths. Social Networks, 32(3):245-251, 2010. 2

[23] Reinhard Diestel. Graph Theory, volume 173 of Graduate Texts in Mathematics. Springer, 5th edition, 2016. 3

[24] M. Rubinov and O. Sporns. Complex network measures of brain connectivity: uses and interpretations. Neuroimage, 52(3):1059-1069, 2010. 2

[25] D. R. White and S. P. Borgatti. Betweenness centrality measures for directed graphs. Social Networks, 16(4):335 - 346, 1994. 15 